\title{
Chewing Gum Containing Citric Acid Reduces the Burden of Periodontal Pathogens
}

\author{
Hiroki Nikawa*,1, Sachiyo Igarashi ${ }^{2}$, Osamu Takasu², Hideo Tataka ${ }^{2}$, Fumiki Harano ${ }^{2}$, Shigeo \\ Shinohara $^{2,3}$, Seicho Makihira ${ }^{1}$, Toshinobu Takemoto ${ }^{1}$, Takeshi Murayama ${ }^{1}$, Takahiro Satoda ${ }^{1}$, \\ Hideaki Amano ${ }^{1}$ and Hidemi Kurihara ${ }^{4}$
}

\author{
${ }^{1}$ Institute of Oral Science, Hiroshima University, Faculty of Dentistry, 1-2-3 Kasumi, Minami-ku Hiroshima, 734-8553 \\ Japan \\ ${ }^{2}$ Otsu Laboratory for Skin Care Science, Rejuvenate Division, Otsuka Pharmaceutical Co., Ltd. 3-31-13 Saigawa, Otsu, \\ Shiga, 520-0002 Japan \\ ${ }^{3}$ Department of Experimental Radiology, Shiga University of Medical Science, Seta Tsukinowa-cho, Otsu, Shiga, 520- \\ 2192, Japan \\ ${ }^{4}$ Department of Periodontology \& Endodontology, Division of Frontier Medical Science, Graduate School of Biomedi- \\ cal Sciences, Hiroshima University. 1-2-3 Kasumi, Minami-ku Hiroshima, 734-8553 Japan
}

\begin{abstract}
Objective: In a preliminary study, we noted that citric acid has the potency to induce human beta defensins (hBD-2) in salivary secretions. As hBDs are thought to ameliorate periodontal disease by suppressing key periodontal pathogens we developed a citric acid-containing chewing gum (CA-gum) to test this hypothesis. Here we report, the effect of CA-gum on three major periodontal pathogens Prevotella intermedia (Pi), Tannerella forsythia (Tf), Treponema denticola $(T d)$ and Fusobacterium species $(F b)$.
\end{abstract}

\begin{abstract}
Methods: A randomized, double-blind, placebo-controlled trial with 61 individuals, was conducted over 14 days. Each subject was allowed to chew CA-gum (31 subjects) or placebo gum (30 subjects) at irregular intervals, but was instructed to eat at least three pieces of gum a day. None had active caries, symptoms of gingivitis or periodontal disease, smoking history, or concurrent medication. To determine the levels of oral carriage of the putative pathogens, saliva was collected from each subject by paraffin chewing in a standard manner and, the number of total cultivative microorganisms (CFUs) determined. Five key periodontal pathogens, $P g, P i, T f, T d$ and $F b$ present in saliva were determined quantitatively using real-time polymerase chain reaction (PCR) (BML Inc., Saitama, JAPAN).

Results: Compared with the placebo chewing gum group the CA-gum group had significantly lower levels of Prevotella intermedia, Tannerella forsythia, and Fusobacterium spp. in their saliva. (Wilcoxon t-test; $\mathrm{p}<0.01$ ). However, chewing of either type of gum did not affect the salivary carriage of total cultivative bacteria (Wilcoxon t-test; $\mathrm{p}>0.05$ ).

Conclusion: Consumption of citric acid-laced chewing gum reduces significantly the burden of periodontal pathogens in saliva.
\end{abstract}

Keywords: Saliva, beta defensin, periodontal bacteria, citric acid.

\section{INTRODUCTION}

Periodontal disease is a common oral disease characterized by a chronic inflammatory process accompanied by destruction of surrounding connective tissue and alveolar bone, and sometimes loss of teeth [1]. The primary causative agents of periodontal disease are particular gram-negative anaerobic bacteria that accumulate in the gingival sulcus.

Bacterial colonization triggers both an innate immunity and host response and acquired immunity. The numerous enzymes and antimicrobial factors present in the saliva and crevicular fluid aid in the neutralization of bacterial components of supragingival and subgingival microbial biofilms. These include lysozyme, lactoferrin, peroxidase, bradykinin,

*Address correspondence to this author at the Institute of Oral Science, Hiroshima University, Faculty of Dentistry. 1-2-3 Kasumi, Minami-ku Hiroshima, 734-8553 Japan; E-mail: hirocky @ hiroshima-u.ac.jp thrombin, fibrinogen, complements, antibodies, and neutrophil-derived components and antibacterial peptides [2-4].

The human beta defensins (hBDs), in particular, are small, cationic, cysteine-rich peptides with potent antimicrobial activity against both gram-positive and -negative bacteria, fungi, and viruses [5], and are produced by epithelial cells of the skin, many lining mucosa, such as intestinal, airway and oral and/or gingival tissues. Among the hBDs, a number of in vitro studies have shown the inducibility of hBD-2 in human keratinocytes exposed to proinflammatory cytokines and components of microorganisms, implying its more specialized role in the innate defense compared with hBD-1, which is less active in disease state and less effective in killing the several microorganisms [6,7].

The expressions of hBDs, particularly $\mathrm{hBD} 2$, in vivo, were reported to play a role in protection from periodontal disease. It is suggested that hBD-1 and -2 were frequently 
expressed in the granular and spinous layers of gingival epithelia and their expression might be associated with periodontal health and disease [8]. In addition, the fact that high levels of hBD2 and 3-mRNA expressed in healthy tissues, as compared with tissues in the status of periodontitis, suggest an important protective role for hBDs in the host immune response to infection by periodontal pathogens [9].

Dunsche et al. have shown that hBDs were expressed widely in oral tissues, not only in epithelial cells but also in salivary glands, such as parotid glands, submandibular glands, small labial glands and sublingual glands [10]. On the other, Fehlbaum et al. has shown that small amino acids including L-isoleucine and several of its analogs can specifically induce epithelial $\beta$-defensin expression [11]. Hence, these findings, taken together, suggested the possibility that the stimuli on the oral tissues particularly salivary glands, by amino acids or acids, could induce the hBD-2 together with salivary flow, which will be expected to be an aid to control oral diseases like periodontal disease.

Both the chewing and acids are beneficial materials to induction of salivary flow, and the two have been combined in an acid-containing chewing gum under the hypothesis that a kind of acid could stimulate the salivary flow together with an increase secretion of hBD-2 which will be expected to reduce the periodontal pathogens. Hence, in the present study, we examined i) the acid potent to induce both salivary flow and hBD-2, and ii) the effects of dairy gum chewing containing acid, on the oral flora and pathogens.

\section{MATERIALS AND METHODS}

\subsection{Participants and Saliva Collection}

Six healthy adult volunteers ( 3 males: 3 females; mean age $37.8 \pm 3.5$ ) took part in the assay, and were able to provide informed consent. To be included, volunteers had to be non-smokers and have no significant oral, dental or systemic diseases; not taking any medication.

Each volunteer rinsed the mouth with water at least 30 minutes before the saliva collection. Then the unstimulated saliva was collected in advance of the stimulated saliva collection as a negative control.

The stimulated saliva was induced by the load of 50 miligrams of powder or $1.0 \mathrm{~mL}$ of $5 \%$ acid solutions which were commonly used for foods, such as, citric acid, DLmalic acid, boletic acid, glutamic acid, cinnamic acid, ascorbic acid, succinic acid, tartaric acid, adipic acid, lactic acid (solution) and acetic acid (solution). Stimulated saliva was collected into a container on ice at intervals of 0-1, 1-2, 2-3 and 5-6 min after the load of each acid to determine the volume of saliva secreted and the concentration of salivary hBD- 2 by enzyme-linked immunosorbent assay (ELISA, see below).

The data were analyzed by one way analysis of variance (ANOVA) and subjected to the Tukey's multiple range test at $1 \%$ level.

\subsection{Determination of hBD-2 Levels in Saliva}

The concentration of salivary hBD-2 was determined by the use of enzyme-linked immunosorbent assay (ELISA). Each saliva sample was diluted appropriately by phosphate- buffered saline supplemented with $10 \%$ calf serum. AntihBD-2 rabbit serum was purchased from Peptide Institute Inc. (Minoh-city, Osaka Japan), and EZ-Link NHS-LCBiotin purchased from PIERCE Biotechnology Inc., (Rockford, IL, USA) were used for biotin-label. Streptavidin and horseradish peroxidase (HRP) conjugate were purchased from Funakoshi Co. Ltd. (Tokyo, JAPAN). Synthesized hBD-2 (BIO PHARM LIMITED, Shizuoka, JAPAN) was used to standardize the concentration of salivary hBD-2.

The data were analyzed by one- way analysis of variance (ANOVA) and Tukey's multiple range test at $1 \%$ level.

\subsection{Effects of Chewing Gum Containing Citric Acid (CA-gum) and Placebo Chewing Gum (Placebo Gum) on Oral Flora and Pathogens}

A total of 61 healthy subjects was divided into two groups. None of subjects had active caries, symptoms of gingivitis or periodontal disease, smoking habit, or any medication. The compositions of CA-gum and placebo gum are summarized in Table 1. The first group comprising 31 subjects (mean age $19.2 \pm 0.4$ ) ate placebo gum, and the second group (30 subjects, mean age $20.4 \pm 0.8$ ) ate CA-gum for a period of two weeks. Each subject was allowed to eat the chewing gum at spontaneous intervals daily, but instructed to eat at least 3 pieces a day. One piece of CA-gum (3.2g) contains $200 \mathrm{mg}$ citric acid.

Table 1. Composition of Placebo Gum and CA-Gum

\begin{tabular}{|c|c|c|}
\hline & Placebo Gum & CA-Gum \\
\hline \hline maltitol & 61.08 & 60.15 \\
\hline gum base & 22.1 & 22 \\
\hline hydrogenated starch hydrolysate & 15 & 11 \\
\hline Citric acid anhydrous & 0 & 6.25 \\
\hline Glycerol & 1.71 & 0.5 \\
\hline Acesulfame potassium & 0.05 & 0.05 \\
\hline Stevia & 0.05 & 0.05 \\
\hline Total & $100(\%)$ & $100(\%)$ \\
\hline
\end{tabular}

Before and after eating each variety of chewing gum, the levels of oral carriage of microorganisms were determined, as follows. The saliva collections were carried out at least 2 $\mathrm{h}$ after lunch. The subjects were asked to chew a piece of paraffin for $10 \mathrm{~min}$, and the saliva produced was expectorated into a plastic container. The number of total cultivative microorganisms was determined with conventional viable counts. Periodontal pathogens released in saliva, such as Porphyromonas gingivalis (P.g.), Prevotella intermedia (P.i.), Tannerella forsythia (T.f.), Treponema denticola (T.d), and Fusobacterium spp. (Fuso.) were determined quantitatively using real-time polymerase chain reaction (PCR) by BML Inc. (Saitama, JAPAN).

Initially, the effects of CA-gum and placebo gum on salivary flow and salivary hBD2 were examined one week before the start of gum-chewing periods with the same divided groups. Each volunteer rinsed the mouth with water at least 


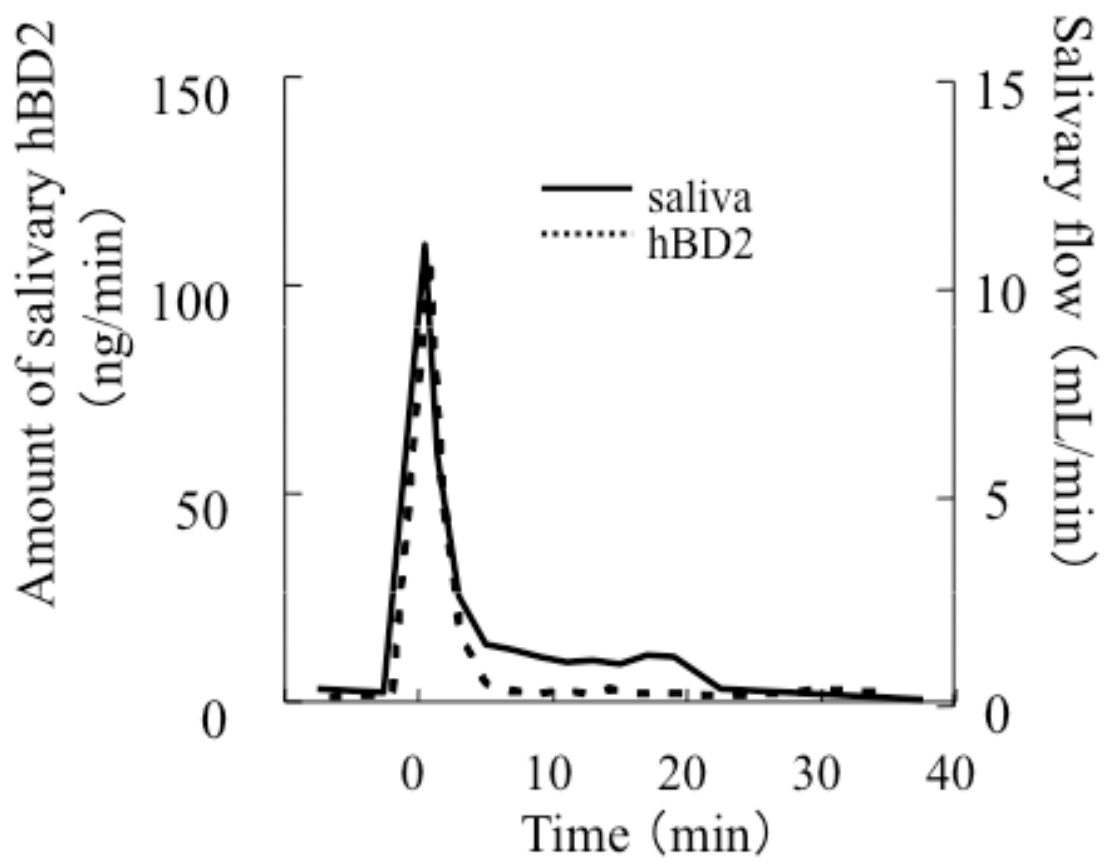

Fig. (1). The mean profile of the changes in the concentration of salivary hBD2 and salivary flow after the load of acid ( $\mathrm{n}=6$ ).

30 minutes before saliva collection. Then the unstimulated saliva was collected in advance the collection of gumstimulated saliva as a negative control. Then the subjects were asked to chew a piece of CA-gum or placebo gum for 1 min, and the saliva produced was expectorated into a plastic container on ice to determine the volume of saliva secreted and the concentration of salivary hBD- 2 by ELISA.

Both the subjects and investigators were unaware of which chewing gum was the CA gum or placebo gum throughout the study. The use of dairy products like Yoghurt or other chewing gum was forbidden for one week prior to and throughout the intervention. The data were analyzed by Wilcxon test at 1 and $5 \%$ levels.

\subsection{Ethical Approval and Informed Consent}

The protocols of saliva sampling and cohort study using chewing gum have been approved by Ethical Authorities at Hiroshima University (22th January 2005). Informed consent was obtained from all the participants after the explanation of both the merits and demerits of the protocol and samples used for the experiment. Because the CA-gum included a high concentration of citric acid, which is known as a causative factor of enamel erosion, we examined the occurrence of dental erosion or troubles in oral health of each participant twice a week, during the periods of cohort tests, to prevent the erosions or other side effects.

All participants were provided the instruction not to take acid flavor foods, beverages or supplements, like chewing gum, candy, pickled Japanese apricot (pickled ume), yogurt and other lactic acid drinks, and/or foods containing lactic acid, during the period of experiment,.

\section{RESULTS}

\subsection{Induction of hBD2 in Saliva by Various Acids}

The amount of hBD2 in saliva rapidly increased with salivary flow after the load of acid (Fig. 1), implying the possibility that some acids were able to induce hBD2 in saliva as expected. Accordingly, which acid is most potent to induce saliva and/or salivary hBD2 was examined. As shown in Fig. (2-a), tartaric acid was most potent to induce saliva, followed by lactic acid, gulutamic acid, cinnamic acid, and reduced in the order of boletic acid, malic acid, ascorbic acid, succinic acid, citric acid, and adipic acid and acetic acid showed the lowest (ANOVA \& Tukey's multiple range test; $\mathrm{p}<0.01)$. The potential of each acid to induce salivary hBD2 was quite different from that to induce saliva (Fig. 2). Citric acid showed the highest potential to induce hBD2 in saliva, followed by malic acid and boletic acid, and tartaric, lactic, succinic, acetic, adipic, ascorbic, gulutamic and cinnamic acid showed the lowest (ANOVA \& Tukey's multiple range test; $\mathrm{p}<0.01$ ), which were not significantly different from induction of hBD2 by water or hBD2 in unstimulated saliva. Thus we have chosen the citric acid to induce the salivary hBD2 hereafter.

\subsection{Effects of Chewing Gum Containing Citric Acid (CA-gum) and Placebo Chewing Gum (Placebo Gum) on Oral Bacteria and Periodontal Pathogens}

As shown in Fig. (3), there were no significant differences observed in either the volume of unstimulated saliva or the concentration of salivary hBD2 between group 1 and group 2. The volume of saliva and the concentration of hBD2 in saliva were significantly increased by chewing either type of gum. The increase in the volume of saliva was significantly higher with the group 2 which ate CA-gum, than those with group 1 (Wilcoxon t-test, $\mathrm{p}<0.01$ ). Similarly, the concentration of salivary hBD2 was significantly higher with group 2 which ate CA-gum, than with group 1 (Wilcoxon t-test, $\mathrm{p}<0.01)$.

The chewing of either type of gum did not affected the oral carriage of total cultivative bacteria (Wilcoxon t-test; $\mathrm{p}>0.05)$. 

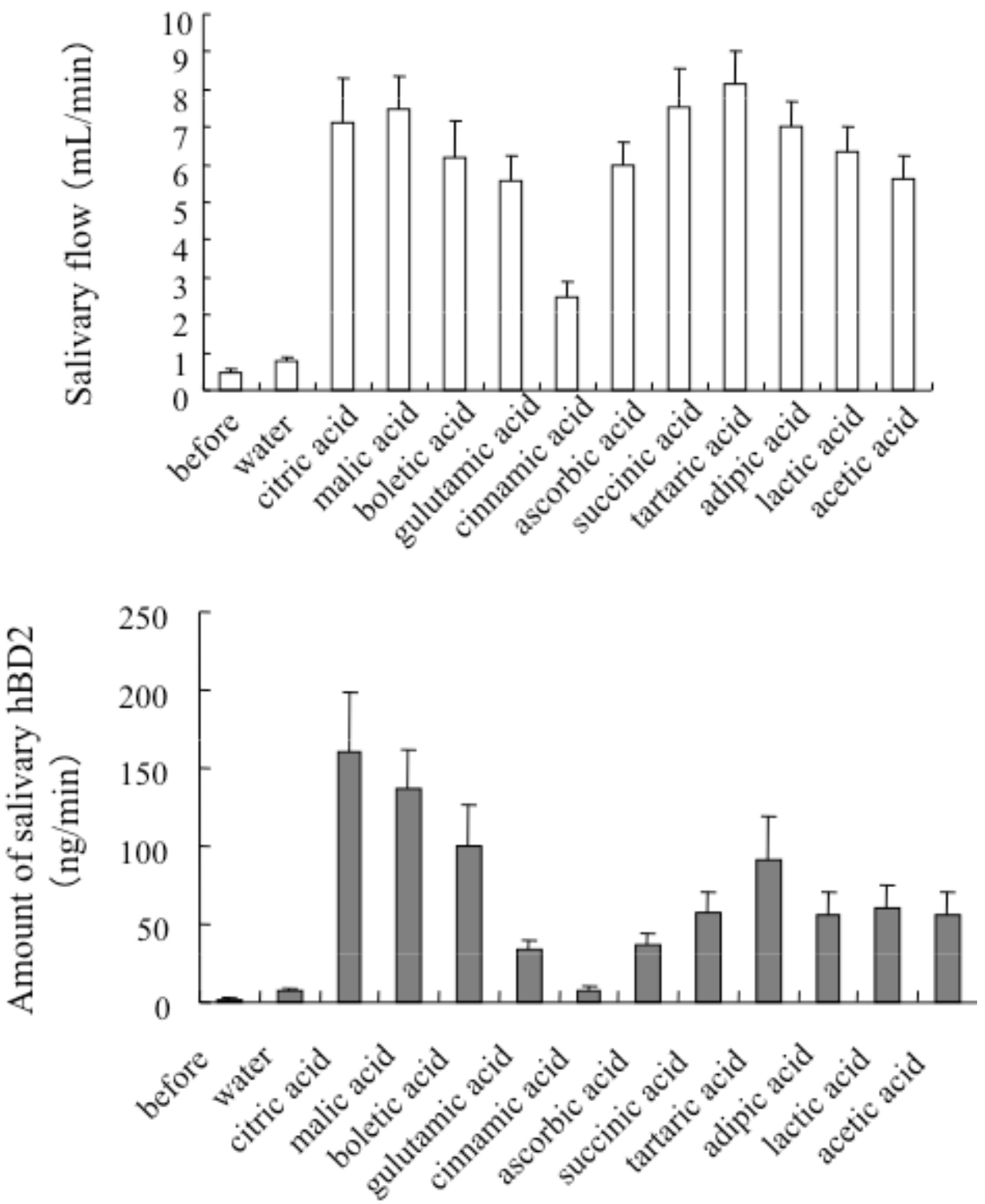

Fig. (2). The potential of acids to induce saliva ( $\square$ ) and concentration of induced salivary hBD2 ( $\mathbf{a})$.

Placebo gum did not significantly reduce the P.g., whereas CA-gum tended to reduce the P.g., but not significantly (Wilcoxon t-test; $p>0.05$ ). CA-gum significantly reduced the oral carriage of P.i. (Fig. 4 ; p $<0.01$ ), but this was not observed with placebo gum (Fig. 4; Wilcoxon t-test; $\mathrm{p}>0.05)$. The oral carriage of T.f. was significantly reduced by CA gum (Fig. 5; p<0.01), and placebo gum also tended to reduce the T.f., but not significantly (Wilcoxon t-test; $\mathrm{p}>0.05)$. Neither gum had significant effects on the oral carriage of T.d., but against Fuso., CA gum reduced significantly (Fig. 6; Wilcoxon t-test; p<0.01).

During the periods of cohort tests, we checked the oral health of each participant and there were no symptom of erosion or side effects caused by routine chewing of CAgum.

\section{DISCUSSION}

\subsection{Induction of hBD2 in Saliva by Various Acids}

In the oral cavity, antimicrobial peptides secreted in saliva or crevicular fluids, such as defensin, histatin, and cathelicidin play a pivotal role as a first-line defense against a succession of invading bacteria [12]. HBDs exert their bactericidal activity by acting on the bacterial membrane, and epithelial cells of the oral mucosal barrier upregulate hBD-2 and hBD-3 upon stimulation by bacterial components or inflammatory mediators $[6,13]$. Thus the induction of saliva should be a beneficial aid for oral health. In addition, we found that the acid would be able to increase salivary hBD2 concentration together with saliva secretion.

Based upon these facts, we firstly examined which acid is most potent to induce both salivary flow and hBD-2, using eleven kinds of acids which are allowed to be incorporated in the foods. The potential of acids to induce saliva were varied depending upon the kinds of acid (Fig. 2). Tartaric acid showed the highest potential, however, the potential of each acid to induce salivary hBD2 was quite different from that to induce saliva (Fig. 2). Citric acid was the most potent to induce hBD2 in saliva, followed by malic acid. Thus we employed citric acid in test gum to induce salivary hBD2. The concentration of salivary hBD2 was significantly increased 

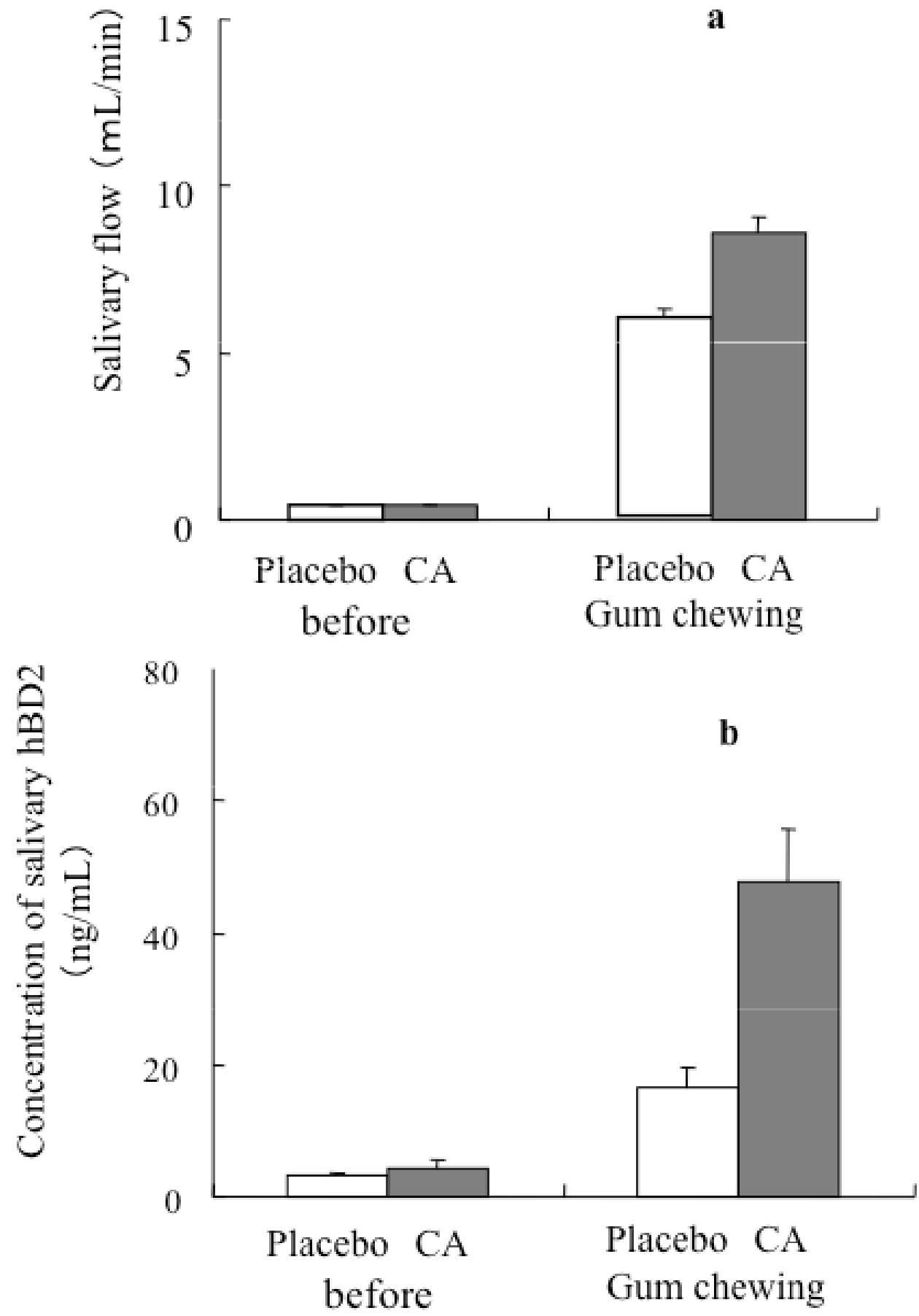

Fig. (3). a) The volume of unstimulated saliva (before) and the volume of stimulated saliva induced by placebo gum ( $\square$; group 1) and CA gum (- group 2). b) The concentration of salivary hBD2 in unstimulated saliva (before) and the concentration of hBD2 in stimulated saliva induced by placebo gum ( $\square$; group 1) and CA gum ( $\mathbf{-}$; group 2).

with CA-gum, compared with placebo gum (Fig. 3), confirming the validity of the incorporation of citric acid, though it should be ideal that the volume of saliva induced by placebo gum is equal to that by CA gum.

\subsection{Effects of Chewing Gum Containing Citric Acid (CA-gum) and Placebo Chewing Gum (Placebo Gum) on Oral Bacteria and Periodontal Pathogens}

The chewing of either type of gum did not essentially affected the oral carriage of total cultivative bacteria (Wilcoxon t-test; $\mathrm{p}>0.05)$. Since more than 500 different kinds of resident bacteria form a normal flora in the oral region exposed to the action of the host defense system [14], it is assumed that commensal bacteria may possess certain mechanisms of escaping the host immune system, and pathogenic bacteria may not [15]. This may be one of the reasons why CA-gum, which induced salivary hBD2, did not affect the total bacterial counts.

It is reported that periodontal diseases are commomly associated with Porphyromonas gingivalis, Prevotella intermedia, Tannerella forsythia, Dialister pneumosintes, Campy- 

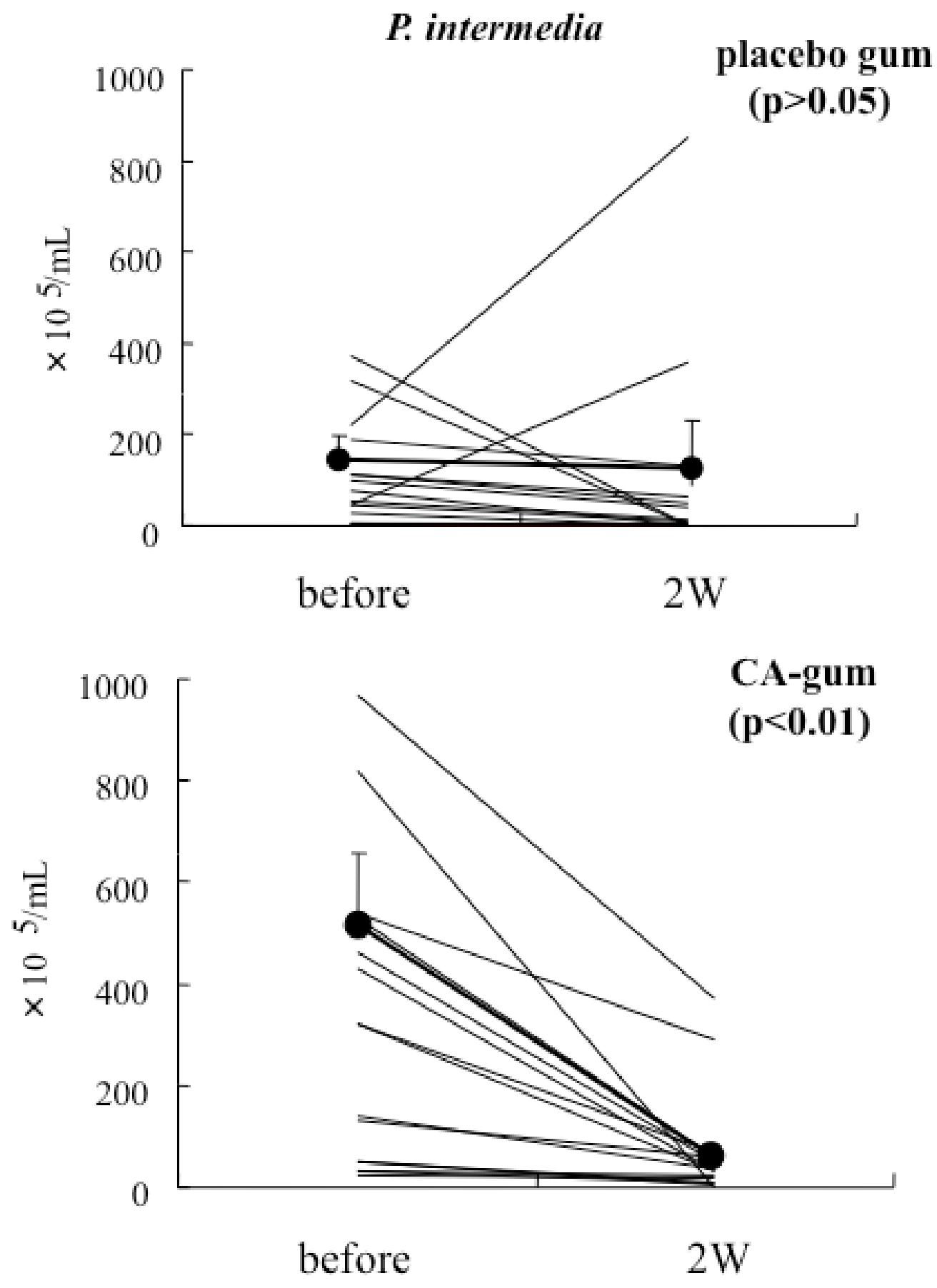

Fig. (4). The effects of continual gum chewing of Placebo gum and CA-gum during 2 weeks on the oral carriage of P.intermedia.

lobacter rectus, Fusobacterium species, Selenomonas sputigena, Peptostreptococcus micros and spirochetes, including Treponema denticola [16]. Thus in the present study, the oral carriage of P.g., P.i., T.f., T.d, and Fuso. were examined as the representative periodontal pathogens.

P.g. is a gram-negative anaerobic bacterium involved in the pathogenesis of chronic adult periodontitis and is frequently found in the subgingival flora of diseased subjects [17]. This organism possesses a variety of virulence factors, including lipopolysaccharides, capsular material, fimbriae and proteases [18]. In the present study, placebo gum did not significantly reduce the P.g., whereas CA-gum tended to reduced P.g., but not significantly (Wilcoxon t-test; $\mathrm{p}>0.05$ ). This was due to the fact that the frequency of the oral carriage of P.g. was very low; 4 of 31 subjects in placebo group and 8 of 30 subjects in CA group, respectively. Further study is necessary to clarify the effects of gum chewing on the oral carriage of P.g.

P.i. is a major periodontal pathogen [19] that is dominant in the periodontal pockets of patients with adult periodontitis $[20,21]$. This bacterium has also been frequently recovered from subgingival flora in patients with acute necrotizing ulcerative gingivitis [22] and pregnancy gingivitis [23]. Recently, it is reported that $P$. intermedia lipopolysaccharide 


\section{T. forsythensis}
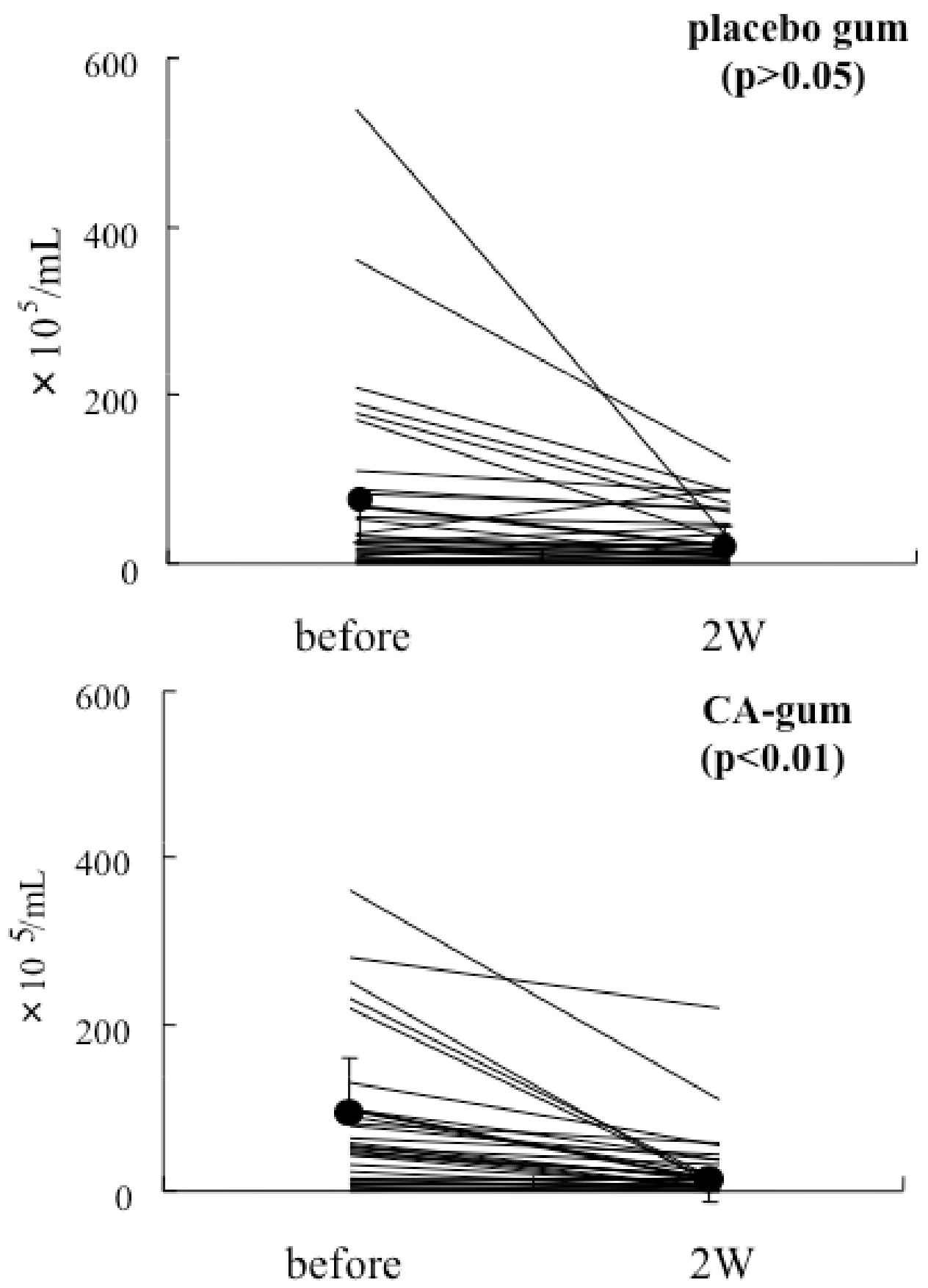

Fig. (5). The effects of continual gum chewing of Placebo gum and CA-gum during 2 weeks on the oral carriage of T. forsythensis.

promotes the production of NOx (nitrogen oxide) which might be important in the pathogenesis of inflammatory periodontal disease [24]. The frequency of the oral carriage of P.i.of each group was 17 of 31 subjects in placebo group and 15 of 30 subjects in CA group, respectively, and CAgum significantly reduced the oral carriage of P.i. (Fig. 6; $\mathrm{p}<0.01$ ), but this phenomenon was not observed with placebo gum (Fig. 4; Wilcoxon t-test; $\mathrm{p}>0.05$ ).

T.f. is well known as a representative periodontal pathogen. It is reported that the metabolic products or a component of T.f. stimulates the growth of P.g. under nutritionlimited conditions and that the interaction between T.f. and
P.g. in growth may be in part related with the synergistic virulence in a periodontitis model [25]. Further, a positive synergy between Fuso. and T.f. in the development of mixed biofilms has been reported [26]. These findings support the importance of T.f. in the development of periodontal disease. In the present study, T.f. was recovered from all participants in either the placebo or CA group. The oral carriage of T.f. was significantly reduced by CA gum (Fig. 5; p<0.01), but placebo gum did not (Wilcoxon t-test; $\mathrm{p}>0.05$ ).

T.d. was found often in deep pockets, and the coinfection of P.g. Aa. and T.d. is reported to cause more serious periodontal destruction than infection of any one or two of the 

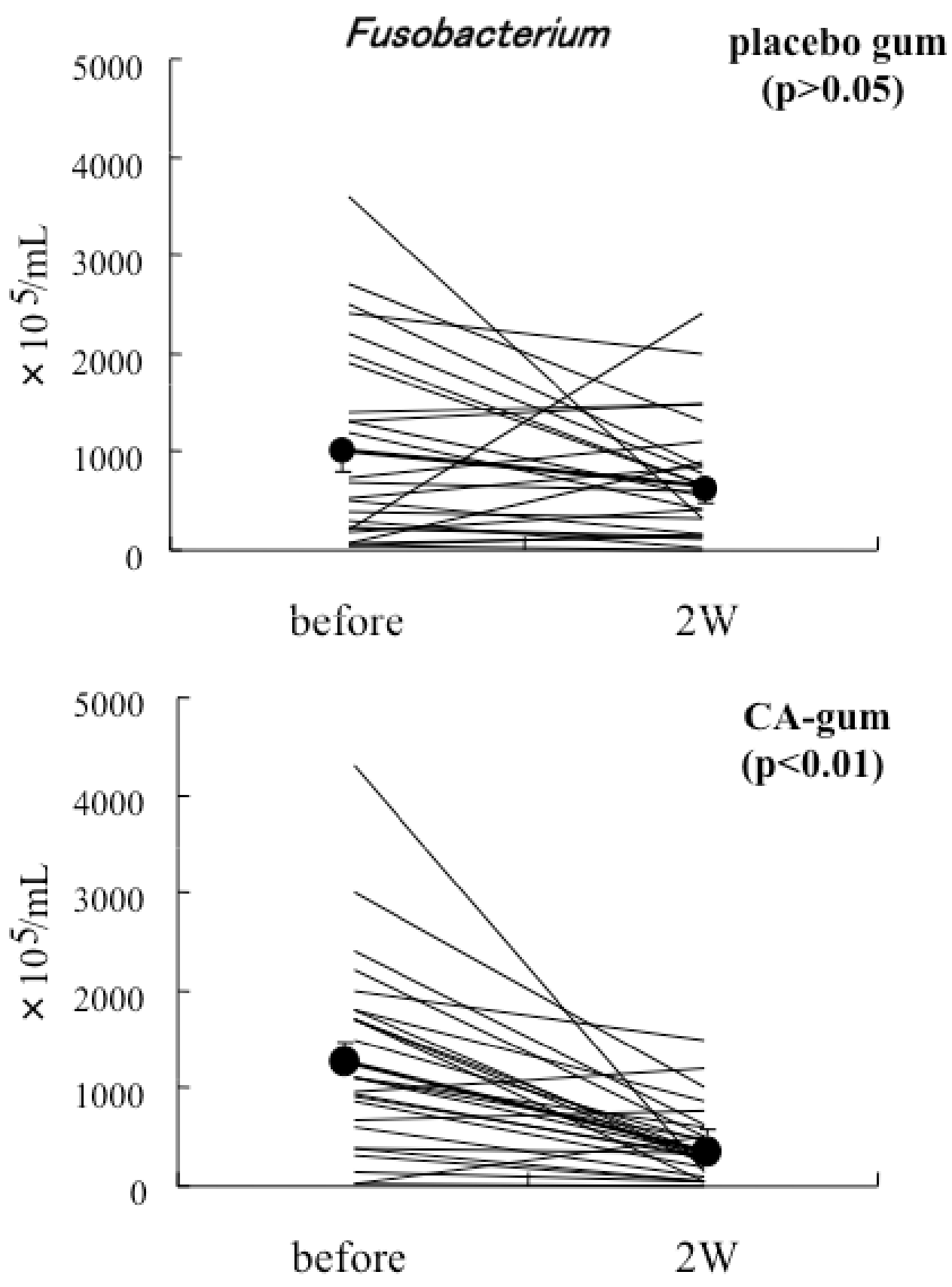

Fig. (6). The effects of continual gum chewing of Placebo gum and CA-gum during 2 weeks on the oral carriage of Fusobacterium spp.

three microbes [27]. The frequency of the oral carriage of T.d. of each group was 21 of 31 subjects in placebo group and 22 of 30 subjects in CA group, respectively, and neither gum was effective to reduce the oral carriage of T.d. The phenomena were not surprising, since it has been recently reported that most strains or isolates of T.d. were resistant to hBD-1, 2 and 3 [28,29].

Fuso. is the most numerous gram-negative bacterium isolated from dental plaque biofilms [18,30]; it is a central species in biofilm development and a pathogen in human infections, including periodontitis. Important properties of Fuso. in biofilm development and pathogenesis include: the abilities to coaggregate to early and late colonizers of plaque biofilms [31,32]; adhere to and invade host tissue cells [33]; induce proinflammatory cytokines [34], and; produce prote- ases $[35,36]$. The frequency of the oral carriage of Fuso. of each group was 25 of 31 subjects in placebo group and 26 of 30 subjects in CA group, respectively, and CA-gum significantly reduced the oral carriage of Fuso. (Fig. 9; p<0.01). This phenomenon was not observed with placebo gum (Fig. 6; Wilcoxon t-test; p>0.05). The reduction of Fuso. by CAgum should be beneficial not only for periodontal health but also for systemic health, since among the bacterial species associated with periodontal disease, Fuso. is the oral organism, most commonly found in systemic infections [30] and is strongly implicated in vaginal infections associated with preterm deliveries [37].

Finally, our results, taken together, suggested that incorporation of citric acid into chewing gum reduced the periodontal pathogens in oral cavity, through increased salivary 
flow and increase in concentration of salivary hBD2, both being the beneficial aids for oral health. In addition, it is suggested that maternal or intrafamilial transmission has been considered a conceivable means of infection in children, and that the saliva is considered to be a major vector of transmission of periodontal pathogens [38]. Thus CA-gum chewing should be expected to be a helpful aid and tool to reduce the familial transmission of periodontal pathogens.

\section{REFERENCES}

[1] Williams RC. Periodontal disease. N Engl J Med 1990; 322: 373381.

[2] Darveau RP, Tanner A, Page RC. The microbial challenge in periodontitis. Periodontol 2000. 1997; 14: 12-32.

[3] Lamkin MS, Oppenheim FG. Structural features of salivary function. Crit Rev Oral Biol Med 1993; 4: 251-259

[4] Schenkels LC, Veerman EC, Nieuw Amerongen AV. Biochemical composition of human saliva in relation to other mucosal fluids. Crit Rev Oral Biol Med 1995; 6: 161-175.

[5] Ganz T. Defensins and other antimicrobial peptides: a historical perspective and an update. Comb Chem High Throughput Screen 2005; 8: 209-217.

[6] Mathews M, Jia HP, Guthmiller JM, Losh G, Graham S, Johnson GK, Tack BF, McCray PB Jr. Production of beta-defensin antimicrobial peptides by the oral mucosa and salivary glands. Infect Immun 1999; 67: 2740-2745.

[7] Krisanaprakornkit S, Kimball JR, Weinberg A, Darveau RP, Bainbridge BW, Dale BA. Inducible expression of human beta-defensin 2 by Fusobacterium nucleatum in oral epithelial cells: multiple signaling pathways and role of commensal bacteria in innate immunity and the epithelial barrier. Infect Immun 2000; 68: 2907-2915.

[8] Lu Q, Jin L, Darveau RP, Samaranayake LP. Expression of human beta-defensins- 1 and -2 peptides in unresolved chronic periodontitis. J Periodontal Res 2004; 39: 221-227.

[9] Bissell J, Joly S, Johnson GK, Organ CC, Dawson D, McCray PBJr, Guthmiller, JM. Expression of beta-defensins in gingival health and in periodontal disease. J Oral Pathol Med 2004; 33: 278285.

[10] Dunsche A, Acil Y, Dommisch H, Siebert R, Schroder JM, Jepsen $\mathrm{S}$. The novel human beta-defensin-3 is widely expressed in oral tissues. Eur J Oral Sci 2002; 110: 121-124.

[11] Fehlbaum P, Rao M, Zasloff M, Anderson GM. An essential amino acid induces epithelial beta -defensin expression. Proc Natl Acad Sci USA 2000; 97 : 12723-12728.

[12] Weinberg A, Krisanaprakornkit S, Dale BA. Epithelial antimicrobial peptides: review and significance for oral applications. Crit Rev Oral Biol Med 1998; 9: 399-414.

[13] Harder J, Bartels J, Christophers E, Schroder JM. Isolation and characterization of human $\beta$-defensin-3, a novel human inducible peptide antibiotic. J Biol Chem 2001; 276: 5707-5713.

[14] Hardie JM, Marsh PD. Streptococci and the human oral flora. Soc Appl Bacteriol Symp Ser 1978; 7: 157-206.

[15] Nishimura E, Eto A, Kato M, Hashizume S, Imai S, Nisizawa T, Hanada N. Oral streptococci exhibit diverse susceptibility to human beta-defensin-2: antimicrobial effects of hBD-2 on oral streptococci. Curr Microbiol 2004; 48: 85-87.

[16] Kamma JJ, Nakou M, Gmur R, Baehni PC. Microbiological profile of early onset/aggressive periodontitis patients. Oral Microbiol Immunol 2004; 19: 314-321.

[17] Nakagawa T, Saito A, Hosaka Y, Ishihara K. Gingipains as candidate antigens for Porphyromonas gingivalis vaccine. Keio J Med 2003; 52: 158-162.

[18] Sundqvist G. Pathogenicity and virulence of black-pigmented gram-negative anaerobes. FEMS Immunol Med Microbiol 1993; 6: 125-137.
[19] Socransky SS, Haffajee AD. The bacterial etiology of destructive periodontal disease: current concepts. J Periodontol 1992; 63: 322331.

[20] Slots J, Bragd L, Wikstrom M, Dahlen G. The occurrence of Actinobacillus actinomycetemcomitans, Bacteroides gingivalis and Bacteroides intermedius in destructive periodontal disease in adults. J Clin Periodontol 1986; 13: 570-577.

[21] Tanner ACR, Haffer C, Bratthall GT, Visconti RA, Socransky SS. A study of the bacteria associated with advancing periodontitis in man. J Clin Periodontol 1979; 6: 278-307.

[22] Chung CP, Nisengard RJ, Slots J, Genco RJ. Bacterial IgG and IgM antibody titers in acute necrotizing ulcerative gingivitis. J Periodontol 1983; 54: 557-562.

[23] Kornman KS, Loesche WJ. The subgingival microbial flora during pregnancy. J Periodont Res 1980; 15: 111-122.

[24] Kim SJ, Ha MS, Choi EY, Choi JI, Choi IS. Prevotella intermedia lipopolysaccharide stimulates release of nitric oxide by inducing expression of inducible nitric oxide synthase. J Periodontal Res 2004; 39: 424-31.

[25] Yoneda M, Yoshikane T, Motooka N, et al. Stimulation of growth of Porphyromonas gingivalis by cell extracts from Tannerella forsythia. J Periodontal Res 2005; 40: 105-109.

[26] Sharma A, Inagaki S, Sigurdson W, Kuramitsu HK. Synergy between Tannerella forsythia and Fusobacterium nucleatum in biofilm formation. Oral Microbiol Immunol 2005; 20: 39-42.

[27] Chen LL, Wu YM, Yan J, Sun WL, Sun YZ, Ojcius D. Association between coinfection of Porphyromonas gingivalis, Actinobacillus actinomycetemcomitans and Treponema denticola and periodontal tissue destruction in chronic periodontitis. Chin Med J (Engl) 2005; 118: $915-21$

[28] Brissette CA, Lukehart SA. Treponema denticola is resistant to human beta-defensins. Infect Immun 2002; 70: 3982-3984.

[29] Brissette CA, Simonson LG, Lukehart SA. Resistance to human beta-defensins is common among oral treponemes. Oral Microbiol Immunol 2004; 19: 403-407.

[30] Moore WEC, Moore LVH. The bacteria of periodontal diseases. Periodontol 2000. 1994; 5: 66-77.

[31] Kolenbrander PE, Andersen RN. Coaggregation of Fusobacterium nucleatum, Selenomonas flueggei, Selenomonas infelix, Selenomonas noxia, and Selenomonas sputigena with strains from 11 genera of oral bacteria. Infect Immun 1989; 57: 3194-3203.

[32] Kolenbrander PE, Andersen RN, Moore LV. Adhere today, here tomorrow: oral bacterial adherence. J Bacteriol 1993; 175: 32473252.

[33] Han YW, Redline RW, Li M, Yin L, Hill GB, McCormick TS Fusobacterium nucleatum induces premature and term stillbirths in pregnant mice: implication of oral bacteria in preterm birth. Infect Immun 2004; 72: 2272-2279.

[34] Huang GT, Haake SK, Kim JW, Park NH. Differential expression of interleukin-8 and intercellular adhesion molecule-1 by human gingival epithelial cells in response to Actinobacillus actinomycetemcomitans or Porphyromonas gingivalis infection. Oral Microbiol Immunol 1998; 13: 301-309.

[35] Bachrach G, Rosen G, Bellalou M, Naor R, Sela MN. Identification of a Fusobacterium nucleatum $65 \mathrm{kDa}$ serine protease. Oral Microbiol Immunol 2004; 19: 155-159.

[36] Mikamo H, Kawazoe K, Sato Y, Tamaya T. Elastase activity of anaerobes isolated from amniotic fluid with preterm premature rupture of membranes. Am J Obstet Gynecol 1999; 180: 378-380.

[37] Hill GB. Preterm birth: associations with genital and possibly oral microflora. Ann Periodontol 1998; 3: 222-232.

[38] Yang EY, Tanner AC, Milgrom P, Mokeem SA, Riedy CA, Spadafora AT, Page RC, Bruss J. Periodontal pathogen detection in gingiva/tooth and tongue flora samples from 18- to 48-month-old children and periodontal status of their mothers. Oral Microbiol Immunol 2002; 17: 55-59. 